\title{
apport des cires de diagnostic et des prothèses d'attente dans les cas prothétiques complexes
}

RÉSUMÉ La décision thérapeutique dans les cas prothétiques complexes est prise à la suite de l'analyse de tous les paramètres répertoriés à partir de l'examen clinique. Elle doit être ensuite matérialisée par des modèles de diagnostic, véritables maquettes de la restauration prothétique terminale et qui vont servir de fil conducteur pendant tout le traitement. De plus, ces maquettes vont également permettre la réalisation des prothèses provisoires. Étape primordiale de la restauration prothétique, la phase des provisoires permet, d'une part de restaurer les fonctions altérées et d'autre part, de tester les configurations occlusales et les choix esthétiques.

Amal BOUZIANE

Spécialiste en parodontologie, C.C.T.D. Rabat,

12 , rue Oued Zem, $n^{\circ} 4$

10000 Rabat.

\section{Salwa BERRADA}

Professeur de l'enseignement supérieur en prothèse adjointe

à la Faculté de médecine dentaire de Rabat.

Neji BENZARTI

Professeur de l'enseignement supérieur, Chef de service de parodontologie au C.C.T.D. Rabat.

\section{MOTS CLÉS}

cire de diagnostic prothèse $d$ 'attente prothèse complexe 


\section{introduction / définition}

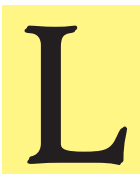

es cires de diagnostic ou wax-up représentent la partie reconstituée par les éléments prothétiques fixés et sont réalisées par la technique de cire ajoutée[1]. Le wax-up permet une correction en cire selon un plan occlusal idéal, pour obtenir des rapports occlusaux et des morpholo- gies coronaires convenables. L'importance des corrections est variable selon les désordres. C'est une véritable proposition thérapeutique faite à l'appareil manducateur du patient, laquelle est ensuite transposée dans les conditions cliniques à travers la prothèse temporaire qui en est l'exact reflet[2].

\section{intérêts des cires de diagnostic}

- intérêt dans le diagnostic

Un diagnostic précis conduit à un choix et à un plan de traitement efficaces et bien fondés. Cependant, une difficulté fondamentale du choix, de la planification, puis de la réalisation du traitement réside dans l'ambiguité de cette notion de traitement pour le patient. Les modèles de diagnostic constituent le support visuel de démonstration indispensable aux explications qui permettent d'identifier des objectifs communs et de gagner la confiance du patient dans le traitement proposé. Ce patient éprouve alors le sentiment qu'il participe activement au traitement[3-7].

L'expression «modèles de diagnostic» ou «cires de diagnostic» trouve sa justification dans le fait que ces maquettes permettent dès le stade de l'analyse initiale de poser un certain nombre d'indications. Un wax-up fonctionnel et esthétique renseigne sur les possibilités de modifications anatomiques et structurelles des dents à reconstituer par la prothèse fixée, dans le respect des principes occlusaux[8].
- Il renseigne le praticien sur la nécessité d'une coronoplastie, d'une dépulpation ou d'un allongement coronaire[9] (fig. 1).

- Il guide le praticien dans le choix du type d'intervention chirurgicale à réaliser.

- Dans le cas de grandes restaurations, les références initiales sont inutilisables. Il faut donc les rétablir puis les conserver jusqu'à l'élaboration prothétique finale. En conséquence, les cires de diagnostic vont préciser le schéma occlusal en redessinant les courbes occlusales frontale et sagittale, en redéfinissant la dimension verticale et l'engrènement. Ce projet se conçoit alors comme une proposition thérapeutique pour l'occlusion de la prothèse [9] (fig. 2 et 3 ).

- En présence de diastèmes entre les dents et lorsque le traitement orthodontique n'est pas envisagé, le waxup permet de fermer les diastèmes et de rétablir des proportions harmonieuses indispensables à la réussite 


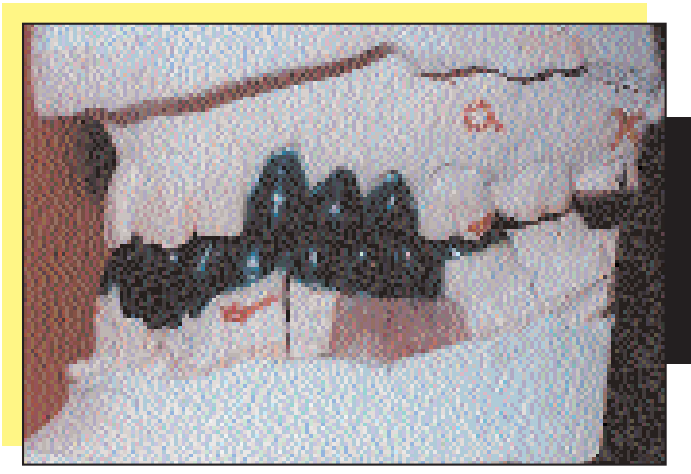

fig. 1 Le wax-up sur ce modèle a permis d'objectiver la nécessité de meuler la cuspide disto-vestibulaire de la 26 et de réaliser une élongation coronaire sur 33 .
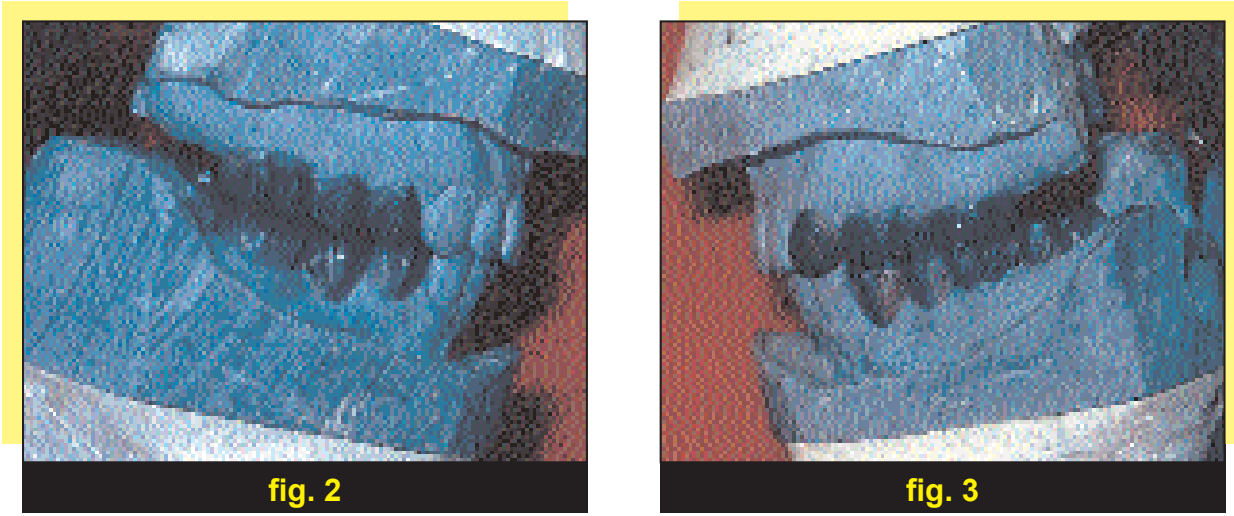

fig. 2 et 3 Cires de diagnostic élaborées sur articulateur rétablissant l'occlusion chez une patiente ayant perdu les références postérieures et ayant une diminution de la DVO. L'élaboration du wax-up permet de recentrer et de caler la mandibule dans le schéma occlusal de fonction canine (Cas Pr S. Berrada).

esthétique de la restauration prothétique.

- À l'opposé, en cas d'encombrement dentaire, le wax-up renseigne sur la possibilité d'une extraction stratégique qui permettra un gain d'espace dans le sens de la largeur. Cette extraction a pour objectif le respect des principes biologiques et esthétiques qui imposent le dégagement des embrasures interdentaires et l'alignement dentaire.
- intérêt dans la thérapeutique pré-prothétique

L'apport des modèles de diagnostic dans la thérapeutique pré-prothétique consiste à aménager le plan de référence occlusal et à réaliser les prothèses provisoires.

\section{AMÉNAGEMENTS OCCLUSAUX PRÉ-PROTHÉTIQUES}

Préalablement à des restaurations prothétiques, vouloir normaliser les rap- 
ports intra et interarcades est justifié pour atteindre des objectifs d'amélioration de la fonction et de l'esthétique.

La préparation occlusale initiale favorise une mise en condition dentaire et neuro-musculo-articulaire permettant d'envisager la réhabilitation prothétique dans de meilleures conditions. Les moyens d'ajustement occlusaux dépassent le meulage sélectif qui visera surtout une meilleure stabilité occlusale plutôt que la création d'un profil d'occlusion idéal. Ces moyens utilisent pour la conception de nouvelles courbes occlusales : la prothèse, l'orthodontie ou la chirurgie orthognathique.

La rupture de la courbe de Spee par édentement précoce et non compensé entraîne l'égression des dents antagonistes qu'il faut soit corriger par un élément prothétique fixé (onlay de recouvrement, couronne céramo-métallique, etc.), soit extraire pour réaménager une courbe postérieure sans interférence ou augmenter l'espace prothétique. Le montage des modèles sur articulateur et la réalisation des cires de diagnostic permettent d'évaluer les possibilités d'intégration des éléments prothétiques dans le système occlusal du patient[10].

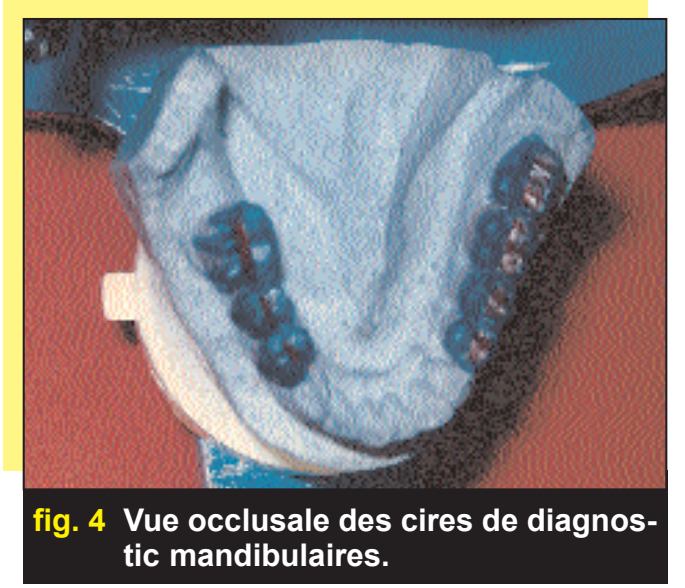

\section{RÉALISATION \\ DE PROTHĖSES PROVISOIRES}

Les restaurations provisoires sont prépondérantes pour la réévaluation fonctionnelle, esthétique, biologique et psychologique. Dans ce souci de réévaluation, une stratégie de traitement est établie selon les différents concepts et données auxquels doit répondre toute réhabilitation prothétique.

Les prothèses provisoires jouent plusieurs rôles :

\section{Stabilisation de I'occlusion[3, 11-14, 16]}

La prothèse provisoire représente une phase primordiale dans la planification de l'anatomie occlusale statique et dynamique de la restauration d'usage. Cette prothèse intérimaire permet à l'appareil manducateur d'assurer ses fonctions normales de mastication, de phonation et de déglutition. La réalisation sur articulateur des maquettes en cire des dents, étape incontournable, permet d'élaborer un schéma occluso-fonctionnel (fig. 4) et de le reproduire sur les restaurations provisoires réalisées en résine acrylique (fig. 5).

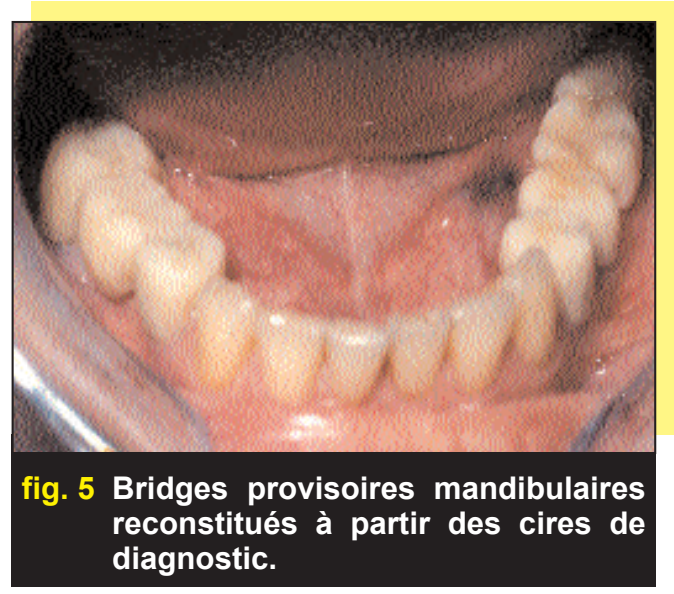



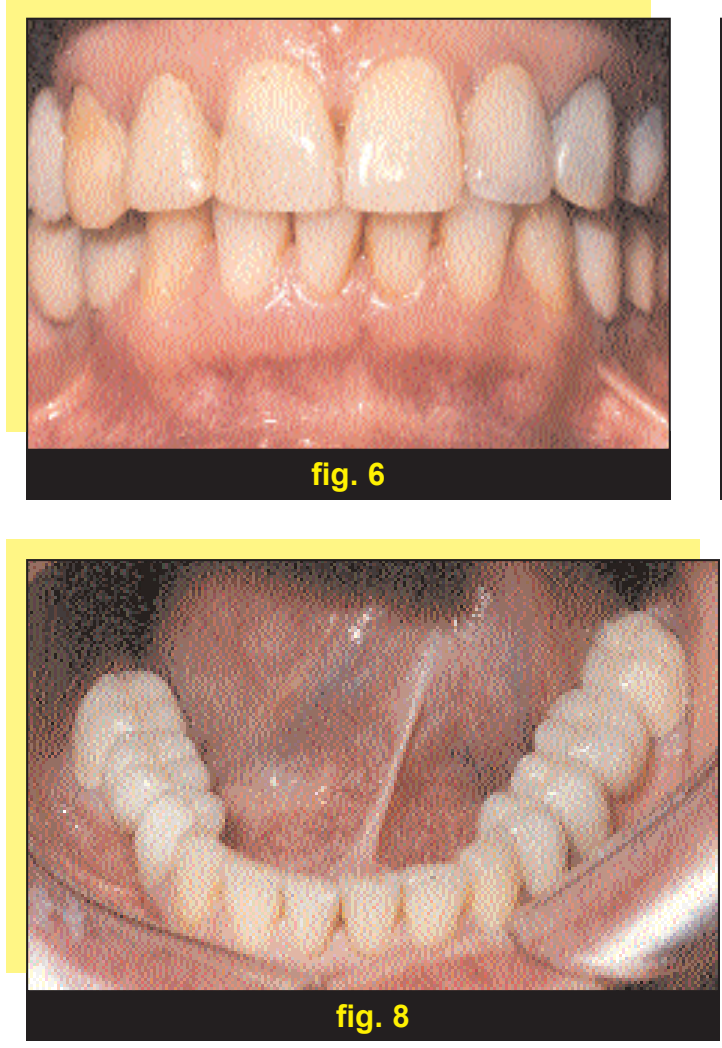

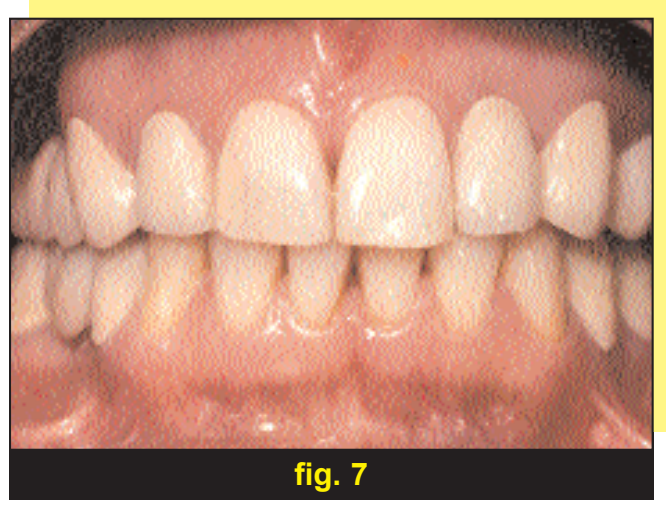

fig. 6 à 8 Reconstitutions fixées permanentes maxillaires et mandibulaires à l'image des prothèses provisoires.
Ces reconstitutions provisoires stabilisent l'occlusion en permettant d'assurer un calage de la mandibule et le positionnement des condyles dans leurs cavités glénoïdes, compatible avec un bon fonctionnement articulaire. Elles permettent aussi d'éviter les égressions dentaires avec leur parodonte, de tester la validité de la proposition thérapeutique et son acceptation par le patient et de confirmer le guide antérieur qui est le facteur le plus important d'une reconstruction occlusale durable.

Après une période appropriée de port de la prothèse provisoire au cours de laquelle la morphologie palatine et la fonction sont harmonisées avec la cinétique mandibulaire du patient, la table incisive est programmée à l'aide du guide personnalisé, ce qui contribuera à la reproduction des particularités palatines et occlusales (morphologie, orientations et trajectoires) sur les prothèses d'usage (fig. $5,6,7$ et 8 ).

\section{Maintien ou restauration de l'esthétique}

Le maintien, l'amélioration ou la restauration totale de l'esthétique concernent un certain nombre d'éléments.

Les modifications sont apportées à la forme, la position, les relations et la couleur des dents détériorées, fracturées ou discolorées. Selon la complexité du cas, une deuxième génération de prothèses provisoires peut être réalisée au laboratoire (provisoires de deuxième intention), afin d'appréhender au mieux le 
résultat définitif[13,15]. De plus, la modélisation des tissus gingivaux inscrite dans un tel cadre ne peut être que favorable au dernier stade du traitement. La restauration finale n'a plus qu'à être la réplique d'une esthétique prothétique testée et intégrée par le patient[16].

L'abrasion des bords libres des incisives peut entraîner l'égression de celles-ci avec l'ensemble du parodonte provoquant un sourire gingival. Le repositionnement du niveau de gencive peut être effectué par gingivectomie, en particulier dans le cas de dents courtes et si la profondeur du sulcus et l'espace biologique le permettent. Dans le but de communiquer le projet esthétique d'aménagement tissulaire au patient et au chirurgien, des facettes en résine acrylique sont réalisées à partir des cires de diagnostic. Elles sont construites en surépaisseur sur des modèles issus des cires et sur lesquels le contour gingival prévu par la chirurgie est dessiné. Elles sont ensuite mises en bouche et le patient peut alors se rendre compte du projet. De plus, cette maquette en résine va servir de guide pour les incisions et les sutures pratiquées lors de la chirurgie[17].

\section{Protection et réparation biologique[ $[13,14,18-20]$}

La prothèse provisoire favorise les mesures d'hygiène bucco-dentaire et facilite l'élimination de la plaque. Elle joue le rôle de guide cicatriciel gingival après la chirurgie parodontale et permet aux tissus gingivaux de retrouver une architecture physiologique. Elle peut mettre en évidence la nécessité de corrections des asymétries ou des irrégularités gingivales qui font suite à l'évolution du contour gingival au fur et à mesure que les tissus retrouvent la santé. Elle assure aussi la protection et la réparation des tissus parodontaux après la préparation prothétique.

La restauration provisoire préserve la santé pulpaire en réalisant une protection étanche de la préparation et assure la protection mécanique, physique et chimique de la préparation quelle que soit la nature de son matériau (dentine, composite, verre-ionomère, alliages) et enfin elle contribue à l'étanchéité des obturations canalaires.

\section{- intérêt dans la thérapeutique prothétique}

La prothèse fixée de moyenne étendue est une reconstruction prothétique qui peut intéresser le secteur antérieur ou les secteurs cuspidés. Les reconstructions de grande étendue concernent quant à elles au moins la totalité d'une arcade. Selon qu'elle est de moyenne ou de grande étendue, les problèmes et objectifs de la reconstruction fixée sont différents[21].

La nécessité d'utiliser un articulateur semi-adaptable s'impose pour rétablir la situation des dents antérieures, du plan d'occlusion et tester une dimension verticale et une relation intermaxillaire adaptées au terrain.

Les prothèses provisoires valident ces éléments. On peut les corriger et ce n'est qu'après une parfaite adaptation et tolérance de ces changements que la restauration globale finale peut être terminée[7, 10, 20, 22, 23].

Les prothèses d'attente, conçues à partir de la maquette diagnostique qu'avait accepté le patient, permettent ainsi aux prothèses permanentes de répondre aux objectifs constructifs suivants : 
- choix et sélection des ancrages[24]; - fixation et maintien de la relation intermaxillaire.

L'enregistrement de la relation intermaxillaire en relation centrée se fait à l'aide des transferts d'occlusion ou avec de la cire Moyco, par sectorisation de la prothèse transitoire[25]: l'enregistrement sur les dents préparées se fera d'un côté, pendant que les provisoires sont en place de l'autre côté et inversement[26].

\section{ÉLABORATION DU SCHÉMA OCCLUSAL CHOISI}

Le schéma occluso-fonctionnel est fortement inspiré de celui établi sur les dents provisoires.

Le concept de protection canine implique, dans le sens sagittal, une courbe de Spee relativement plate et haute postérieurement. Une accentuation de la courbe d'occlusion peut entraîner, dans ce cas, des interférences occlusales postérieures. De même, dans le plan frontal, la version vestibulaire des prémolaires et molaires maxillaires et la version linguale des prémolaires et molaires mandibulaires faiblement accentuées permettent une courbe de Wilson relativement plate pour éviter les interférences non travaillantes[9].

Si la protection de groupe existait déjà ou si la canine ne remplit pas son rôle protecteur ou si la situation parodontale n'est pas favorable, la protection de groupe est indiquée. Il est nécessaire en général de réduire la pente des versants cuspidiens et d'ouvrir légèrement la concavité palatine des incisives et canines[27]. La prise en charge travaillante intéresse, si possible, la canine, les prémolaires et la première molaire dont aucune ne doit avoir une prépondérance sur l'autre. Sinon le résultat obtenu est une interférence travaillante.

\section{REPRODUCTION DU GUIDE ANTÉRIEUR[12, 28-31]}

Plusieurs éléments anatomiques doivent être pris en considération concernant le guidage antérieur. Plus le plan d'occlusion est horizontal, plus la pente incisive devra être importante, plus la hauteur et la pente cuspidienne des dents postérieures sont importantes, plus la pente incisive devra être importante et enfin plus les courbes de compensation sont marquées, plus la pente incisive devra être importante. Le réglage ou le façonnage de la table incisive est nécessaire pour que le prothésiste dispose des informations indispensables à la reconstruction du guide antérieur.

\section{TRANSFERT \\ DES PARAMÈTRES ESTHÉTIQUES AU LABORATOIRE[32, 33]}

Lors d'une réhabilitation $\mathrm{du}$ bloc antéro-supérieur, les informations d'ordre esthétique (position de la ligne médiane, longueur et position des dents antéro-supérieures par rapport aux lèvres supérieure et inférieure) doivent être transmises au laboratoire. Un contre-montage d'un modèle des restaurations provisoires permet de transférer les paramètres esthétiques. Une empreinte est donc réalisée avec les couronnes provisoires en place et permet la réalisation d'un modèle. Ce dernier est monté en articulateur par rapport au modèle de l'arcade antagoniste, et les repères peuvent être modifiés en fonction des besoins. 


\section{élaboration et utilisation des cires de diagnostic}

\section{- élaboration}

La réalisation des cires de diagnostic passe par des préalables occlusaux. L'examen occlusal, partie intégrante de l'examen clinique, comporte l'observation plus spécifique de l'unité dentaire, de l'orientation et la continuité des courbes occlusales de Spee et de Wilson, de la stabilité de l'OIM et de la liberté des mouvements mandibulaires de toute interférence sans résistance musculaire.

À ce stade d'analyse, le montage des moulages d'étude sur l'articulateur après enregistrement de la relation centrée, facilite l'examen et donne la possibilité de mieux apprécier les rapports occlusaux du patient[10].

C'est au cours de l'analyse occlusale, que l'articulateur révèle tout son intérêt[34-36]. L'articulateur, par sa tige incisive, est le seul et unique instrument permettant de matérialiser les modifications de dimension verticale d'occlusion en dehors de la bouche. La mesure de la dimension verticale d'engrènement (DVE) sur articulateur matérialise la dimension verticale d'intercuspidation maximale du patient. Après estimation de la dimension verticale de la relation centrée (DVRC), la différence DVRC DVE représente la quantité de dimension verticale qu'il faudrait perdre au cours de l'équilibration pour retrouver la dimension verticale d'occlusion existante.

Les modèles sont fractionnés et montés sur articulateur. Ce qui permet, dans un premier temps, de faire abstraction artificiellement des secteurs cuspidés. L'analyse du guide antérieur met alors en évidence le type de schéma occlusal à adopter après avoir supprimé les prématurités existant dans la relation intermaxillaire choisie. La technique de Filastre qui consiste à retirer les secteurs cuspidés mandibulaires et relever la tige incisive, représente un bon moyen pour l'analyse occlusale du secteur antérieur ce qui permet d'éliminer tout contact prématuré postérieur. Dans un deuxième temps, la recherche des interférences sera envisagée au cours des différents mouvements fonctionnels.

L'analyse occlusale, loin de compliquer la tâche du praticien, simplifie le traitement. Elle aboutit à la réalisation d'un prototype morphologique des futures prothèses, les cires de diagnostic.

Sur les modèles d'étude montés sur articulateur, les éléments dentaires concernés par une restauration prothétique fixée sont meulés. Quelques pertuis sont réalisés et enduits d'une fine couche de cire collante permettant une bonne adhésion de la cire ajoutée de reconstruction[37].

La restauration débute par les dents antérieures lorsqu'elles sont concernées par les besoins prothétiques. Les incisives mandibulaires, d'abord, puis les incisives maxillaires en respectant la situation optimale du bord libre, la forme du cingulum et la pente incisive. Suivent ensuite les canines[2]. La dernière étape consiste à finir la surface occlusale et créer les sillons secondaires[1]. 
- utilisation

Plusieurs techniques sont proposées pour conserver, transmettre, et utiliser les références obtenues. Les cires de diagnostic sont élaborées sur un premier jeu de modèles montés en articulateur. L'empreinte des arcades reconstituées en cire est ensuite réalisée à l'aide d'un silicone. Les moules ainsi obtenus peuvent être utilisés directement en les repositionnant, chargés de résine, sur un second jeu de modèles dont les éléments à restaurer ont été arbitrairement réduits. On obtient alors des coques de résine représentant un profil occlusal idéal. Dans le cas de restaurations provisoires de longue étendue, on peut déposer un fil métallique de renfort ou une tresse de matériau synthétique dans l'empreinte en silicone, puis $y$ couler un dégradé de résines choisies à partir d'une teinte de base appropriée[2].

Les dents provisoires ainsi obtenues permettent de rétablir l'esthétique et la fonc- tion. Ces deux critères peuvent être améliorés à tout moment, soit par soustraction de résine, soit par addition jusqu'à ce que le résultat soit parfaitement intégré. Toutes les excursions mandibulaires sont ainsi contrôlées pour valider ou améliorer le concept occluso-prothétique établi avec les cires de diagnostic. Un jeu de modèles représentant les arcades restaurées par les provisoires est monté en articulateur. Le glissement en propulsion suivant les inclinaisons palatines des dents, permet de transférer les informations concernant le guide antérieur au niveau du plateau incisif de l'articulateur. Lorsque l'empreinte des dents supports a été réalisée, il est possible de monter le modèle de travail obtenu par la technique d'enregistrement alterné (mordu alterné) qui permet de conserver toutes les données inter-arcades. On retrouve ainsi, lors de la mise en place de la prothèse d'usage, tous les critères définis lors de l'élaboration des cires de diagnostic et validés par les provisoires[34, 38-40].

\section{cas clinique}

L'apport des modèles de diagnostic dans les restaurations prothétiques de longue étendue est illustré à travers un cas clinique suivi au centre de consultation et de traitement dentaires de Rabat.

\section{- observation clinique}

Monsieur M. A. âgé de 48 ans, fonctionnaire, se plaint de l'absence de ses incisives centrales mandibulaires et de la mobilité des incisives latérales. L'examen dentaire (fig. 9, 10 et 11) révèle l'absence de $36,31,41$ et 46 , une nécrose pulpaire de la 15 qui est fortement délabrée et une mobilité de la 12 due à un trauma occlusal (effet de coin exercé par 43 sur son antagoniste lors $\mathrm{du}$ mouvement de fermeture). L'examen parodontal montre la présence d'une parodontite chronique avec des récessions presque généralisées.

L'examen de l'occlusion indique un inversé d'articulé au niveau des secteurs moyens en rapport avec une endoalvéolie maxillaire et l'égression 

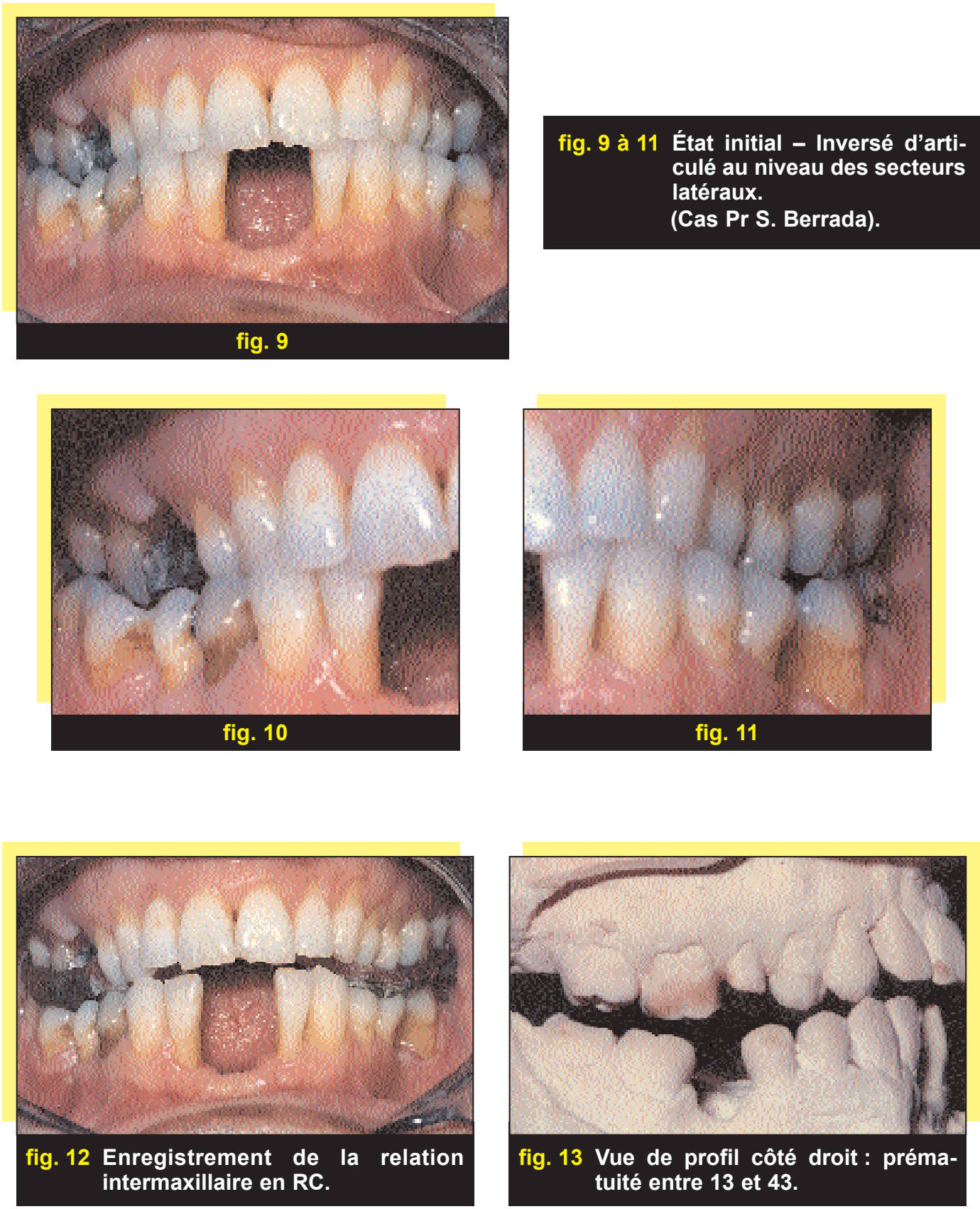

importante du bloc incisivo-canin inféL'examen des modèles d'étude montés rieur à l'origine d'un plan d'occlusion haut situé et d'un rapport couronne clinique/racine clinique défavorable. sur articulateur au moyen d'une cire de centrée met en évidence une prématurité entre 13 et 43 (fig. 12 et 13). 
- décision thérapeutique

Le plan de traitement comporte une reconstruction fixée totale de l'arcade mandibulaire avec un bridge monolithique de 34 à 44, un bridge de 35 à 37 , et un bridge de 45 à 47 .

La correction prothétique de l'endoalvéolie maxillaire passe par la réalisation d'un bridge de 13 à 16 (remplace la 15 extraite) ainsi que des couronnes unitaires sur 23, 24 et 25. Une correction orthodontique de l'endoalvéolie n'est pas envisagée à cause des récessions généralisées et du parodonte fin du patient.

\section{- réalisation prothétique}

Le modèle mandibulaire est préparé selon la technique de Filastre qui consiste à retirer les secteurs cuspidés mandibulaires pour analyser le guide antérieur. Un wax-up est réalisé au niveau du secteur antéro-mandibulaire et des deux canines maxillaires pour situer le plan incisivo-canin mandibulaire à un niveau plus physiologique. Après obtention d'une butée antérieure

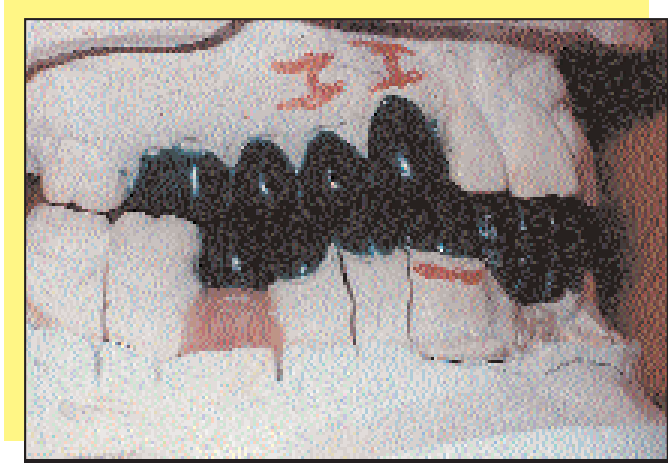

fig. 14 Modèles de diagnostic côté droit. stable, on remet secondairement les segments latéraux fractionnés et on finit d'élaborer les cires de diagnostic postérieurement, dans le schéma de protection canine. Le wax-up (fig. 14 et 15) a permis d'objectiver la nécessité d'extraire la 32 et la 42 très égressées, de meuler la cuspide disto-vestibulaire de la 26 , de reconstituer 13,14 et 25 au moyen d'inlay-cores pour rectifier leur position palatine, de reconstituer la 33 par inlay-core pour corriger sa position vestibulaire, et de poser l'indication d'une élongation coronaire pour les canines inférieures (fig. 16 et 17).

Le traitement pré-prothétique comporte l'assainissement des lésions parodontales, l'extraction de 32 et 42 , des traitements endodontiques sur $16,14,13$, $23,24,25,37,34,35,33,43,44$, et 45 et une gingivectomie au niveau des canines mandibulaires.

Le traitement prothétique est ensuite réalisé par reconstitutions corono-radiculaires sur 13, 14, 25 et 33 (fig. 18), pose de prothèses provisoires (fig. 19), et pose des bridges permanents maxillaires et mandibulaires (fig. 20, 21 et 22).

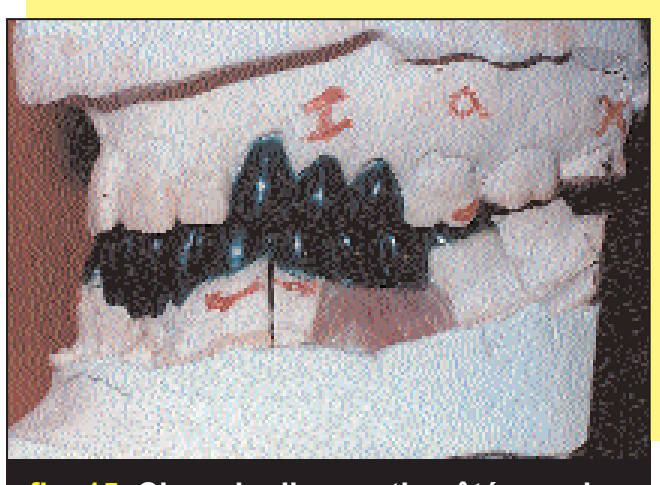

fig. 15 Cires de diagnostic côté gauche. 


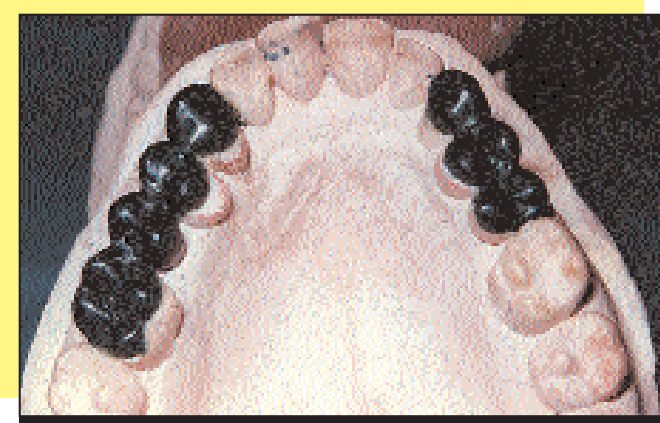

fig. 16 Correction de la position palatine de 13,14 et 25 par le wax-up.
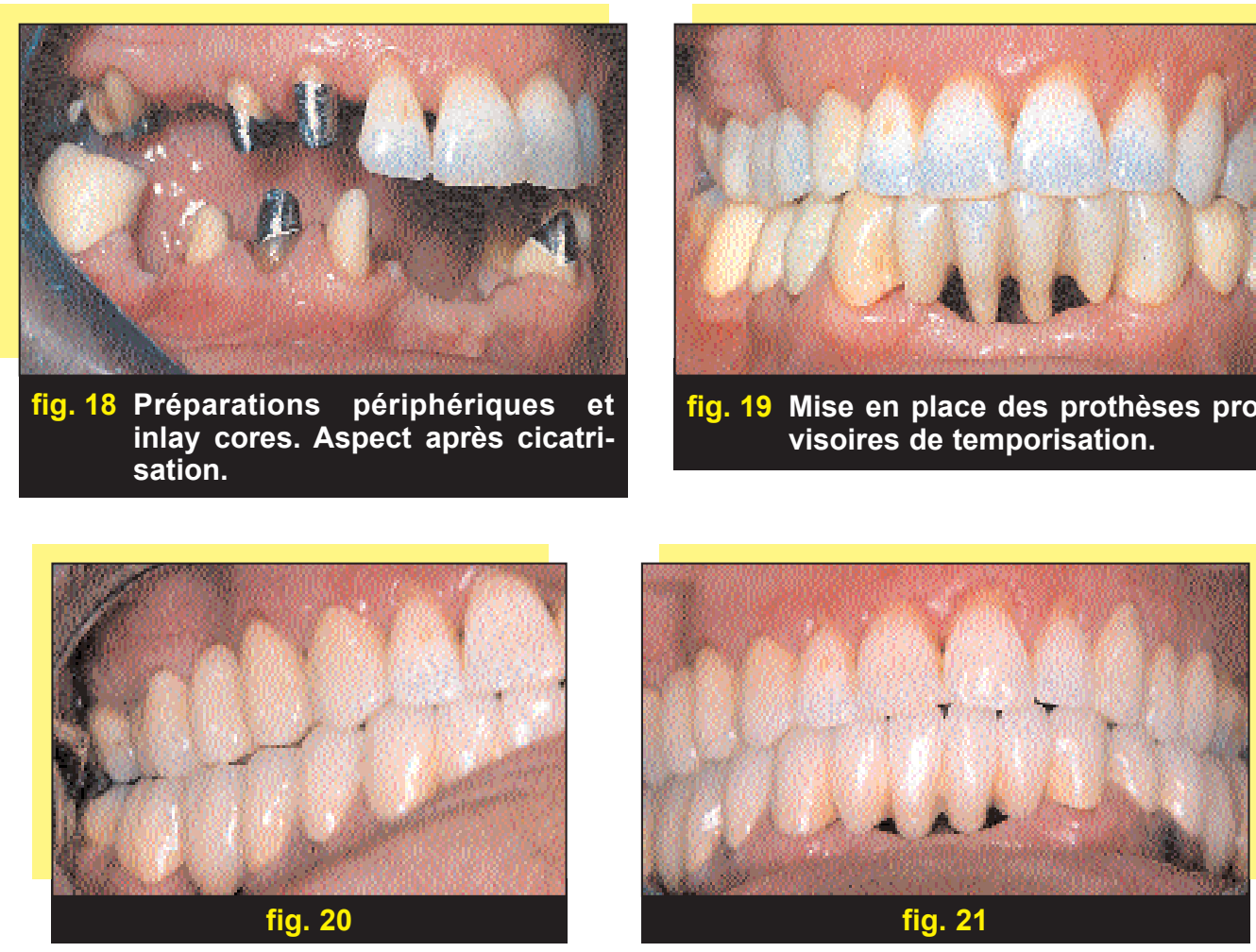

fig. 20 à 22 Mise en place des bridges permanents.

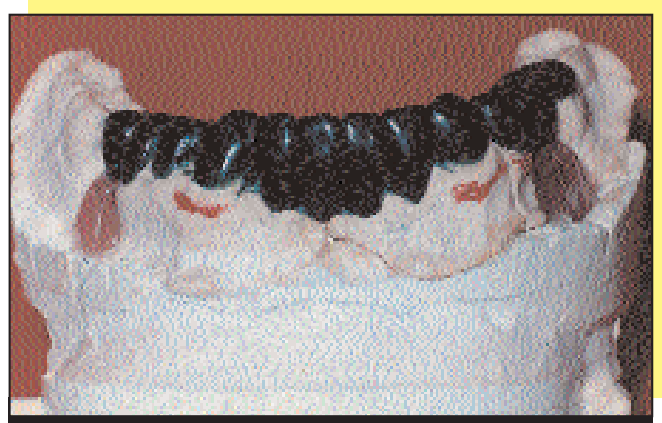

fig. 17 Matérialisation du plan d'occlusion optimal par le wax-up.

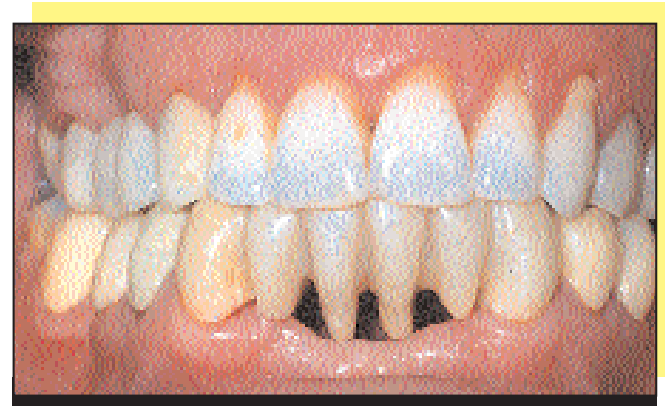

fig. 19 Mise en place des prothèses provisoires de temporisation.

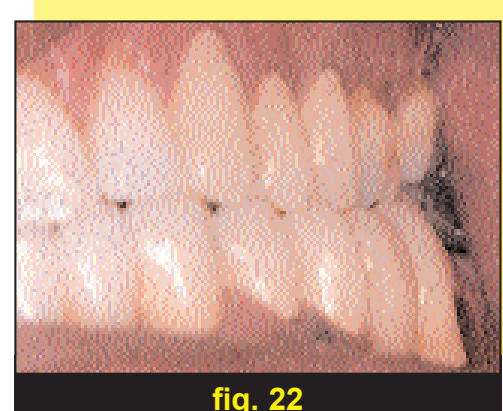

fig. 22 


\section{conclusion}

Les reconstitutions prothétiques de moyenne et de grande étendue, imposent des étapes qui doivent se succéder selon un enchaînement logique sans nécessiter de retour en arrière au cours $\mathrm{du}$ traitement. La prothèse qui constitue le dernier stade du plan de traitement, doit faire appel à la même démarche clinique et l'utilisation des modèles de diagnostic est un guide constant tout au long de sa réalisation. La réalisation des cires de diagnostic facilite l'établissement de la thérapeutique prothétique en évitant les erreurs de conception.

\section{bibliographie}

1. Lefèvre $M$. Relations cliniquelaboratoire en prothèse composite.

Réal Clin 1998;9(4):465-480.

2. Thepin JC, Ravalec $X$ La fonction thérapeutique de la prothèse provisoire fixée dans les grandes restaurations.

Cah Prothèse 1998;104 29-49.

3. Carlier JF, Schitly J.

Le plan de référence occlusal en prothèse partielle amovible. Inf Dent 1992;8:555-564.

4. Valentin $\mathrm{CM}$.

Du plan au programme de traitement : méthodes et stratégie.

Réal Clin 1995;6(1):7-23.

5. Serruya G.

Traitement des

édentements unilatéraux postérieurs.

Réal Clin 1998;9(4):455-463.

6. Lquarti $G$, Comte B, Allard $Y$, Martin JP, Bois D.

Temps pré-prothétiques

de la réalisation

des bridges.

Encycl Méd Chir,

Odontologie, 23-272-A-10, 1999, 8p.

7. Smaniotto P, Berti CE Réhabilitation du secteur antéro-mandibulaire : les critères essentiels. Art et Techniques Dentaires 2000;11(1):37-44.

8. Borie JF, Estrade D, Fleiter B Harmonisation pré-prothétique des contacts occlusaux. Réal Clin 1993;4(2):153-161.

9. Garnier B

Les modèles de diagnostic : cires et montages directeurs en prothèse. Intérêt, élaboration, utilisation.

Inf Dent 1989;42:4121-4129.

10. Turlay C.

Situations occlusales pré-prothétiques : pourquoi et comment intervenir?

Cah Prothèse 2000;112:7-23.

11. Radeani $M$, et al. Restauration d'une occlusion fonctionnelle et de l'esthétique. Rev Int Parodont Dent Rest 1992;12(1):62-71.

12. Valentin $C$, Yakhou $O$. Reconstruction du guide antérieur par des artifices de prothèse conjointe. Réal Clin 1993;4(2):163-176.

13. Dowek D.

Utilisation d'un

articulateur : pourquoi, quand, comment ? Réal Clin 1993:4(2):135-152.
14. Colombier ML, Taeib T, Bigot C.

La préparation tissulaire pré-chirurgicale. Réal Clin 2000;11(2):149-158.

15. Dersot JM, Martin D. Rétablissement du sourire. Un abord parodontoprothétique réfléchi. Inf Dent 1998;42:3407-3413.

16. Magne $P$, Magne $M$. Patient, clinicien et céramiste : une triade vers l'esthétique. Inf Dent 1994;76(42):3963.

17. Spear F, Kovich V. Perfection esthétique dans le secteur antérieur. Inf Dent 1994;42:3964-3966.

18. Unger $F$, Lemaître $P$, Hoornaert A.

Guide clinique : prothèse fixée et parodonte. Paris : CdP, 1997.

19. Laborde G, Borguetti A. Préparations inta-créviculaires et parodonte marginal. Réal Clin 1992;3(2):203-214.

20. Kosinski S.

Processus décisionnel en chirurgie parodontale pré-prothétique. Réal Clin 2000;11(2):159-168.

21. Belser Urs C, Wiscott HW. Objectifs de traitement 
en prothèse fixe.

Cah Prothèse 1996;96:6-12

22. Galindo D, Sollys JL,

Graser GN.

Long term reinforced

fixed provisional

restorations.

J Prosthet Dent 1998;79(6) 698-701.

23. Arcidiacono $A$, Marzola $R$

Derbabian K.

Un système

de communication entre

le prothésiste dentaire

et le chirurgien-dentiste.

Art et Techniques Dentaires 2000;11(2):83-89.

24. Difebo $G$.

Aspects particuliers dans le cadre des grandes restaurations fixées : choix, acquisition et maintien de la position de référence. Inf Dent 1997;8:504-506.

25. Malquarti $G$, Pirel $C$, Bois $D$. Atlas des cas cliniques en prothèse fixée. Encycl Méd Chir, Odontologie, 23-272-D-10, 1997, 18p.

26. Sarfati $E$.

Examen clinique et décision thérapeutique en prothèse.

Actual Odontostomatol 1997:198:425-441.
27. Lubespère $A$, Jacquet $E$, Crétot M.

Le guidage antérieur en prothèse fixée. L.Q.O.S. 1986;11:25-46.

28. Laurent M, Orthlieb JD. Approche occlusale d'une restauration des incisives maxillaires. Cah Proth 1997:99:10-19.

29. Dupas PH Approche simplifiée de l'occlusion ou réalité clinique quotidienne? Art et Techniques Dentaires 2000;11(1):53-58.

30. Moulin P, Doukhan JY, Kleinfinger $\mathrm{S}$.

Les étapes pré-prothétiques. Réal Clin 1995;6(4):411-421.

31. Renault $P$, Pierrisnard $L$. Occlusion et prothèse fixée : démarche décisionnelle et principes du plan de traitement. Cah Proth 2000;112:63-82.

32. Crottaz C, Wiscott HWA Enregistrement et transfert de la relation intermaxillaire.

Cah Proth 1995;96:107-128.

33. Sabek M, Trevelo A. Restauration du guide antérieur, impératifs et moyens techniques.
Rev Odonto Stomatol 1994; 4:281-307.

34. Lefèvre $M$, Vincent $G$. Transmission des données prothétiques de la clinique au laboratoire. Réal Clin 1993;4(2):211-220.

35. Voiry JG, et al. Réhabilitation occlusale prothétique.

Encycl Méd Chir 23-265-C-10, 1998, 8p.

36. Dupas $\mathrm{PH}$.

Les articulateurs semi-adaptables. Comment ? Pourquoi ? Quand?

Vélizy : CdP, 1996.

37. Lefèvre $M$, Vincent $G$, L'official, Vincent M. Modèles de diagnostic. $2^{e}$ partie : réalisation. Cah Proth 1989;67:26-37.

38. Sarfati E, Radiguet J. Les schémas occlusaux en prothèse fixée. Cah Proth 1997;100:37-50.

39. Marmy O, Cioppi G, Michelini F. Restaurations provisoires. Cah Proth 1996;96:44-52.

40. McMaster DE. Using the laminar impression technique for provisional restorations. JADA 2000;131:1324-1325.

\section{SUMMARY}

\section{Advantage of wax-up and interim prosthesis in complex prosthesis cases}

A. BOUZIANE, S. BERRADA, N. BENZARTI

The therapeutic decision of complex prosthetic cases is made after an analysis of all the identified parameters from clinic examination. It is then confirmed using wax-up, actual scale models of the final restoration that will serve as the central reference throughout the treatment.
These models will also help in the production of interim prosthesis. The temporary phase of treatment, which is of primary importance, allows the restoration of altered functions, and the testing of occlusal configurations and aesthetic decisions.

keywords: wax-up, temporary prosthesis, complex prosthesis. 